Para enlazar con este artículo / To link to this article:

http://dx.doi.org/10.14198/fem.2017.29.05

Para citar este artículo / To cite this article:

Gallego-Morón, Nazareth. «Más allá de las estadísticas. Invisibilización y negación de la discriminación de género en la Universidad». En Marcos Jesús Iglesias Martínez e Inés Lozano Cabezas (coords.), La (in)visibilidad de las mujeres en la Educación Superior: retos y desafios en la Academia. Feminismo/s, 29 (junio 2017): 125-152, DOI: 10.14198/fem.2017.29.05

\title{
MÁS ALLÁ DE LAS ESTADÍSTICAS. INVISIBILIZACIÓN Y NEGACIÓN DE LA DISCRIMINACIÓN DE GÉNERO EN LA UNIVERSIDAD ${ }^{1}$
}

\section{BEYOND STATISTICS. INVISIBILIZATION AND DENIAL OF GENDER DISCRIMINATION AT UNIVERSITY}

\author{
Nazareth GALLEGO-MORÓN \\ Universidad Pablo de Olavide \\ nazareth.gallego@gmail.com \\ orcid.org/0000-0002-8172-4515
}

\section{Resumen}

Las cifras estadísticas muestran la existencia de una doble discriminación de género en la Universidad en Argentina: la segregación horizontal y vertical (techo de cristal). Además, la revisión de la literatura confirma la invisibilización y baja concienciación por parte del cuerpo docente universitario. El presente trabajo forma parte de una investigación más amplia sobre la participación de mujeres y hombres en la Universidad Nacional de Salta (Argentina). El principal objetivo consiste en analizar la existencia o no de discriminación de género en la Universidad y profundizar en las percepciones que el profesorado posee acerca de ésta y de las barreras y obstáculos existentes. Para ello, en primer lugar, se ofrece un análisis descriptivo de la participación de hombres y mujeres en el sistema universitario argentino. En segundo lugar, se presentan los resultados de una encuesta aplicada al cuerpo docente de la Universidad Nacional de Salta. Éstos confirman una escasa percepción del problema. Además, esta negación es mayor entre los hombres. La existencia de redes informales de poder es señalada como la principal barrera para la promoción por ambos sexos.

1. Este trabajo de investigación ha sido financiado por la Universidad Pablo de Olavide (Sevilla) en el marco del Proyecto GENDER AND CITIZENSHIP (GENDERCIT). Subprograma People del 7. a Programa Marco Europeo de I+D en el marco del Programa Marie Curie Action. FP7-PEOPLE-2012-IRSES N. ${ }^{\circ} 318960$. 
Palabras clave: discriminación de género, segregación ocupacional, techo de cristal, Universidad, Argentina.

\begin{abstract}
The statistical figures show the existence of a double gender discrimination in Argentine university: horizontal and vertical occupational segregation (glass ceiling). In addition a review of the literature confirms the invisibilization and low awareness by the university teaching staff. This paper is part of a larger research on the participation of women and men at the National University of Salta (Argentina). The main objective of this article is to analyze the existence or not of gender discrimination at university and to deepen the perceptions of teachers have about it and the existing barriers and obstacles. For this, in the first place, a descriptive analysis of the participation of men and women in the Argentine University System. Second, the results of a survey applied to the total teaching staff of the National University of Salta are developed. These confirm a scant perception of the problem. Moreover, this denial is greater among men. The existence of informal power networks is singled out as the main barrier to advancement by both sexes.
\end{abstract}

Keywords: gender discrimination, occupational segregation, glass ceiling, university, Argentina. 


\section{LAS MUJERES EN EL MERCADO DE TRABAJO EN ARGENTINA}

En los últimos 20 años se ha producido un avance considerable con respecto a la incorporación de las mujeres en el mercado de trabajo en América Latina y el Caribe. La participación femenina en la fuerza laboral pasó de un 44,5\% en 1995 a un 52,6\% en 2015 (OIT) como consecuencia de un mayor acceso de las mujeres a niveles educativos más altos y a una serie de transformaciones socioculturales -particularmente el control sobre la maternidad-, junto con la existencia de medidas de conciliación, como la subvención de servicios para el cuidado de la infancia (Busso y Romero).

Sin embargo, como muestra un informe elaborado en 2016 por la Organización Internacional del Trabajo (OIT), los progresos realizados por las mujeres en el mundo educativo no se han traducido en una posición más favorable en el trabajo. De este modo, la tasa de desempleo de las mujeres, para el año 2015, era más alta que la de los hombres tanto a nivel mundial (5,5\% para los hombres y 6,2\% para las mujeres) como en la región de América Latina y el Caribe, donde la diferencia porcentual era aún mayor: 5,4\% de hombres desempleados frente al $8,1 \%$ de mujeres.

La participación de las mujeres en el mercado de trabajo argentino comenzó a aumentar en la década de los sesenta, y, en especial, a partir de 1990 debido a la pérdida de empleo masculino en un contexto de recesión económica (PNUD 20). Tal y como se desprende de las estadísticas, el trabajo de las mujeres ha pasado a convertirse en el principal sustento económico de muchas familias, debido a diversas causas como la existencia de hogares monomarentales, la situación de desempleo o subocupación de los hombres, o la insuficiencia de un único salario. De esta forma, se ha producido un notable crecimiento en las últimas décadas, elevándose en más de diez puntos el porcentaje de mujeres activas desde 1990, cuando representaban el 36,8\% (CTIO).

A pesar del avance que se ha producido, sigue existiendo una situación de desigualdad laboral de género. Al mayor riesgo de desempleo se le suma la precariedad que caracteriza el trabajo femenino: temporalidad, parcialidad, subocupación, baja cualificación, trabajos informales e inseguros, segregación ocupacional (horizontal y vertical) y brecha salarial de género, etc. 


\subsection{Tasa de desempleo, actividad y empleo}

En Argentina, según los últimos datos disponibles de la Encuesta Permanente de Hogares (EPH) proporcionados por el Instituto Nacional de Estadística y Censos (INDEC), la tasa de desempleo nacional se situó en el 8,5\%, en el último trimestre del año 2016. En el caso de las mujeres, el 9,2\% estaban desempleadas frente al $8 \%$ de los varones, lo que llevaba a una brecha de género del orden de 1,2 puntos (Tabla 1).

Tabla 1. Tasa de desempleo de la población de 14 años y más, por sexo. Total 31 aglomerados urbanos. 3. ${ }^{\circ}$ Trim. 2016

\begin{tabular}{|c|c|c|}
\hline Tasa de desempleo total & Tasa de desempleo hombres & Tasa de desempleo mujeres \\
\hline $8,5 \%$ & $8 \%$ & $9,2 \%$ \\
\hline
\end{tabular}

Fuente: Elaboración propia. EPH. INDEC

Durante el tercer trimestre del año 2016, la tasa de actividad argentina era del $57,7 \%$ (Tabla 2). Con respecto al género, existe una brecha considerable de 22,5 puntos: $47,2 \%$ para las mujeres y del $69,7 \%$ para los hombres (INDEC 2016). Esto es consecuencia de una histórica división sexual del trabajo que "parece haber sido universal en toda la historia humana» (Hartmann 255) dentro de una sociedad heteropatriarcal, en la que se han asignado a hombres y mujeres diferentes tareas según la pertenencia a un sexo u otro.

Tabla 2. Tasa de actividad de la población de 14 años y más, por sexo. Total 31 aglomerados urbanos. 3. ${ }^{\circ}$ Trim. 2016

\begin{tabular}{|c|c|c|}
\hline Tasa de actividad total & Tasa de actividad hombres & Tasa de actividad mujeres \\
\hline $57,7 \%$ & $69,7 \%$ & $47,2 \%$ \\
\hline
\end{tabular}

Fuente: Elaboración propia a partir de los datos del EPH. INDEC

Tradicionalmente se han dividido las actividades en función de las características biológicas de las personas, dando lugar a una dicotomía sexual entre el espacio privado/reproductivo -donde las mujeres llevaban a cabo las tareas domésticas y de cuidados no remuneradas-, y el espacio público y productivo -donde los hombres realizaban el trabajo remunerado-. Se trata de una división sexual del trabajo que aún a día de hoy persiste (Torns y Recio 185). Todo ello en base a una teórica complementariedad entre sexos fruto de la especialización y distribución eficiente de tareas (Sarasúa y Gálvez), en la que las 
mujeres han sido consideradas como el sexo débil, quedando relegadas a una situación de «dependencia» respecto a los hombres (Gálvez, Rodríguez, Agenjo y Domínguez 18). Se constituye la lógica del modelo «hombre proveedor de ingresos-mujer ama de casa» (modelo male breadwinner), un contrato social/ sexual (Pateman) según el cual las mujeres deberían satisfacer las necesidades de los varones para que éstos pudieran cumplir con su condición de ciudadano y trabajador asalariado.

La inexistente corresponsabilidad por parte de los hombres da lugar a una menor disponibilidad de tiempo, lo que repercute directamente en la reducción de oportunidades para acceder al mercado de trabajo remunerado (Gálvez y Rodríguez). Como se desprende de los datos proporcionados por las encuestas sobre trabajo no remunerado y uso del tiempo, las mujeres argentinas emplean cada día tres horas más que los hombres para la realización del trabajo doméstico no remunerado, lo que comprende quehaceres domésticos, apoyo escolar y cuidado de personas (INDEC 2014). Sigue habiendo una diferencia de participación en este trabajo de 31 puntos porcentuales a cargo de las mujeres ( $57,9 \%$ los hombres y $88,9 \%$ las mujeres).

Con respecto a la tasa de empleo, ésta se encontraba en el 52,8\% (Tabla 3). Si atendemos a la diferencia por sexos, al igual que ocurriese con la tasa de actividad, la tasa de empleo masculina superaba notablemente a la femenina. Así, el porcentaje de trabajadoras se situaba en el último trimestre del año 2016 en un 42,9\%, mientras que los hombres alcanzaban el 64,1\% (INDEC 2016).

Tabla 3. Tasa de empleo de la población de 14 años y más, por sexo. Total 31 aglomerados urbanos. 3. ${ }^{\circ}$ Trim. 2016

\begin{tabular}{|c|c|c|}
\hline Tasa de empleo total & Tasa de empleo hombres & Tasa de empleo mujeres \\
\hline $52,8 \%$ & $64,1 \%$ & $42,9 \%$ \\
\hline
\end{tabular}

Fuente: Elaboración propia a partir de los datos del EPH. INDEC

\subsection{Segregación ocupacional horizontal}

Analizando la distribución de hombres y mujeres por ramas de actividad, según datos del Boletín de Género realizado por el Observatorio de Empleo y Dinámica Empresarial (OEDE), como se observa en la Tabla 4, para el segundo trimestre del año 2014, las mujeres se agrupaban principalmente en dos ramas de actividad. Por un lado, en el sector servicios, donde representaban el 46,4\% del total de trabajadores/as, y, por el otro, en comercio, con un 34,8\%. Éstas aparecen representadas principalmente en aquellas actividades correspondientes a 
los servicios como es el caso de la enseñanza (73,6\%), los servicios sociales y de salud $(71,2 \%)$ y, los servicios comunitarios, sociales y personales $(45,4 \%)$. Se trata de un conjunto de trabajos que se configuran como una extensión de las tareas y funciones de bienestar y cuidado que tradicionalmente han sido desarrolladas por las mujeres. De este modo, como mantienen Moser y Young, habitualmente las mujeres «trabajan en determinadas ocupaciones cuando tienen algún parecido estructural con su rol familiar» (57).

No obstante, si prestamos atención a la población trabajadora masculina encontramos que su presencia era casi total en la construcción (94,1\%), área fuertemente masculinizada donde prácticamente no trabajan mujeres $(5,9 \%)$ y aquellas que lo hacen realizan trabajos de servicios como la limpieza dentro de este sector. En segundo lugar, su participación sigue siendo mayoritaria entre las actividades primarias $(89,6 \%)$, como la explotación de minas y canteras $(90,9 \%)$ y la agricultura y ganadería $(89,3 \%)$. De igual modo, en el área de la electricidad, gas y agua, el porcentaje de hombres era considerablemente más alto que el de mujeres ( $82,9 \%$ y $17,1 \%$, respectivamente) al igual que sucede en la industria ( $81,4 \%$ de los hombres y $18,6 \%$ de las mujeres).

Tabla 4. Empleo asalariado registrado, privado, por ramas de actividad y sexo. $2 .^{\circ}$ Trim. 2014

\begin{tabular}{|l|c|c|}
\hline Rama de actividad & Mujeres & Hombres \\
\hline Actividades primarias & 10,4 & 89,6 \\
\hline Industria manufacturera & 18,6 & 81,4 \\
\hline Electricidad, gas y agua & 17,1 & 82,9 \\
\hline Construcción & 5,9 & 94,1 \\
\hline Comercio & 34,8 & 65,2 \\
\hline Servicios & 43,4 & 56,6 \\
\hline
\end{tabular}

Fuente: Elaboración propia a partir de los datos del Boletín de Estadísticas de Género y Mercado de Trabajo. OEDE

Esta situación se denomina segregación ocupacional horizontal y hace alusión a la agrupación de hombres y mujeres en ocupaciones y sectores diferenciados, marcados e influenciados por arraigados estereotipos y roles de género (Monreal y Martínez). De este modo, se considera que «hay trabajos remunerados femeninos, cuyo ejercicio es adecuado para las mujeres, mientras que otros son impropios de ellas» (Gómez 123). 
Como consecuencia, el número de empleos a los que las mujeres pueden acceder se ven reducidos, mientras que se encuentran sobrerrepresentadas en otros. Además, aquellos desarrollados principalmente por éstas se caracterizan por una menor valoración y remuneración (Cohen y Huffman). De este modo, en el segundo trimestre de 2014, las trabajadoras argentinas del sector privado cobraban el $76,3 \%$ del salario medio mensual de los hombres (\$9.863 mensuales las mujeres y $\$ 12.922$ los hombres), produciéndose una brecha salarial de género del 23,7. Esta situación se configura como un problema continuo en el tiempo para los/as argentinos/as, puesto que la brecha apenas ha disminuido con el paso de los años, así, en el primer trimestre del año 2012 se encontraba en el 23,8 (OEDE).

\subsection{Segregación ocupacional vertical: el techo de cristal}

A la situación de discriminación horizontal se le suma la discriminación ocupacional vertical, entendida como la escasa presencia de mujeres en las categorías laborales más altas. Se trata del fenómeno del «techo de cristal» (glass ceiling), metáfora utilizada para referirse al conjunto de barreras y obstáculos invisibles, acotados y sólidos que las mujeres deben superar para acceder a los altos cargos y puestos de responsabilidad de la pirámide organizacional y que afecta a las mujeres como grupo, «debido a que son mujeres» (Morrison, White y Van Velsor 13).

Analizando los datos proporcionados por el OEDE, podemos concluir que las mujeres argentinas apenas acceden a las categorías más altas. Como se observa en la Tabla 5, del total de trabajadoras registradas, únicamente el 2,6\% ocupaban los puestos de dirección de las empresas privadas. En el caso de los hombres el porcentaje se elevaba hasta el 3,4\%. En los cargos intermedios, la participación de hombres continuaba siendo mayor que la de las mujeres, 6,8\% y $4,7 \%$, respectivamente. Sin embargo, es en la escala básica, la de ejecución directa, en la que se produce la mayor diferencia porcentual entre sexos. De este modo, el 92,7\% de las mujeres se agrupaban en esta categoría, superando a los hombres en casi tres puntos porcentuales $(89,8 \%)$.

Tabla 5. Composición del empleo según estructura jerárquica, por sexo. 2. ${ }^{\circ}$ Trim. 2014

\begin{tabular}{|l|c|c|c|}
\hline & Directivos/as & Jefes/as intermedios/as & Ejecución directa \\
\hline Mujeres & 2,6 & 4,7 & 92,7 \\
\hline Hombres & 3,4 & 6,8 & 89,8 \\
\hline
\end{tabular}

Fuente: Elaboración propia a partir de los datos del Boletín de Estadísticas de Género y Mercado de Trabajo. OEDE 
Se trata del fenómeno de los «suelos pegajosos» (sticky floor), el cual guarda relación directa con el techo de cristal, y que hace referencia a la acumulación de las mujeres profesionales en las escalas más bajas de las organizaciones (Baxter y Wright). En palabras de Gallego «metafóricamente, parece que las mujeres caminen sobre un suelo que las atrae y les impide continuar avanzando» (65) a lo largo de la estructura jerárquica.

Cabe esperar que el ámbito público, en general, y la Universidad, en particular, se constituyan como espacios de trabajo regidos única y exclusivamente por la meritocracia. Trabajos donde el acceso y los procesos de promoción se rijan por criterios claros y objetivos, basados en la capacidad y méritos del cuerpo docente, alejada de la situación de discriminación de género, que como hemos desarrollado en los párrafos anteriores, las mujeres argentinas encuentran en el sector laboral privado.

Sin embargo, tal y como numerosos/as autores/as e informes institucionales han mostrado (Baylin; Baxter y Wright; Comisión Europea; Fernández; García de León; Jackson y O'Callaghan; Probert; Ogbogu) en la mayor parte de los países del mundo, a pesar de que la participación de las mujeres en la Educación Superior universitaria es mayor que la de los hombres, éstas acceden en menor proporción a la cúspide de la pirámide organizacional y se agrupan en aquellas áreas y campos considerados tradicionalmente femeninos.

En este aspecto reside el interés para la elaboración del presente estudio, con el objeto de realizar una aproximación a la realidad de los y las docentes universitarias argentinas y profundizar en las percepciones que ambos poseen acerca de la discriminación de género en la Academia. Para ello, en primer lugar, se ofrece un análisis descriptivo de la participación de hombres y mujeres en el Sistema Nacional Universitario Argentino. En segundo lugar, se presentan los resultados de una encuesta aplicada al cuerpo docente de la Universidad Nacional de Salta.

\section{METODOLOGÍA}

\subsection{Objetivos}

El presente artículo presenta un doble objetivo. (1) Se pretende conocer la situación de las mujeres en el Sistema Nacional Universitario Argentino, determinando la existencia o no de discriminación de género horizontal y/o vertical. Para ello se realiza un estudio descriptivo a través del análisis de fuentes estadísticas secundarias acerca de la participación de hombres y mujeres (tanto 
entre el alumnado como entre el profesorado) en las diferentes áreas y categorías académicas universitarias.

(2) Se realiza un análisis exploratorio y se profundiza en las percepciones del cuerpo docente de la Universidad Nacional de Salta (UNSa) acerca de la existencia de discriminación de género en el ámbito universitario y de las barreras y obstáculos de género, partiendo de sus propias experiencias personales y profesionales. La bibliografía internacional muestra una baja visibilización del problema por parte del profesorado, incluso por parte de las propias protagonistas, las docentes e investigadoras. Por ello, se indaga sobre esta cuestión y se profundiza en si se producen o no diferencias según el sexo, así como si éstas son significativas. El instrumento utilizado ha sido la encuesta.

\subsection{Muestra}

La UNSa se constituye como una de las universidades más feminizadas del territorio argentino. De acuerdo a los datos proporcionados por el Departamento de Estadísticas Universitarias de la UNSa, en el año 2015 estaba formada por un total de 2.260 personas, de las que las mujeres representaban el $54 \%$, porcentaje superior a la media nacional (48,9\%). Del total de mujeres, el $66 \%$ formaban parte del cuerpo auxiliar docente mientras que el $34 \%$ eran profesoras (62\% y $38 \%$ de hombres, respectivamente). De este modo, además de una alta participación femenina se produce, a priori, una situación de equilibrio con respecto a la participación en los cargos académicos más altos, el del profesorado asociado y titular. Por un lado, el total del profesorado asociado estaba constituido por un $50 \%$ de mujeres y un $50 \%$ de hombres. Por otro lado, en la categoría de titular, el 48,7\% eran mujeres y el 51,3\% eran hombres.

Aunque bien es cierto que en el caso de esta universidad la brecha de género es menor que en el panorama nacional, también lo es que la participación de las docentes va disminuyendo paulatinamente desde el inicio de la carrera hasta los cargos y puestos de responsabilidad y poder, mientras que el de los hombres aumenta continuamente, produciéndose el fenómeno del «tubo que pierde» (leaky pipeline). De igual modo, éstas se agrupan en mayor medida entre los puestos bases de auxiliares, lo que manifiesta la existencia de mayores dificultades para promocionar debido a la existencia de los suelos pegajosos. Además, se produce una situación de segregación horizontal, ejerciendo en áreas tradicionalmente feminizadas, como es la Facultad Ciencias de la Salud, donde representaban el 81\% del total de docentes, o, la Facultad de Humanidades, con el 69,4\% del total (UNSa). 
La muestra de personas que han respondido a la encuesta no es aleatoria. Ésta fue enviada a la totalidad del cuerpo docente conformado por 2.260 personas (1.220 mujeres y 1.040 hombres), durante los meses de julio, agosto y septiembre de 2016, de la que se recibieron 92 respuestas. De éstas, el 59,8\% $(n=55)$ eran mujeres y el 27,2\% ( $n=25)$ hombres. El restante 13\% ( $n=12)$ no reveló su sexo. Con respecto a la edad media de las personas participantes, ésta fue de 46,4 años (46,9 para las mujeres y 43,9 para los hombres). Por otro lado, los/as encuestados/as pertenecían a diferentes áreas académicas y categorías, tal y como se recoge en la tabla 6:

Tabla 6. Área académica y categoría profesional de la muestra

\begin{tabular}{|c|c|c|c|c|c|}
\hline & \multirow{2}{*}{ Frecuencia } & $\%$ & \multicolumn{3}{|c|}{ Sexo } \\
\cline { 4 - 6 } & & & Mujeres & Hombres & Nd \\
\hline Área Académica & & & & \\
Salud & 4 & 4,3 & 1 & 1 & 2 \\
Experimentales & 15 & 16,3 & 7 & 5 & 3 \\
Sociales & 6 & 6,5 & 4 & 1 & 1 \\
Humanidades & 26 & 28,3 & 21 & 4 & 1 \\
Ingenierías & 39 & 42,4 & 22 & 12 & 5 \\
Nd & 2 & 2,2 & - & 2 & - \\
Total & 92 & 100 & & & \\
Categoría Académica & & & & & \\
Profesorado Titular & 6 & 6,5 & 1 & 3 & 2 \\
Profesorado Asociado & 10 & 10,9 & 9 & 1 & - \\
Profesorado Adjunto & 37 & 40,2 & 23 & 8 & 6 \\
Jefe/a Trabajos Prácticos & 30 & 32,6 & 15 & 11 & 4 \\
Auxiliar de Segunda & 1 & 1,1 & - & 1 & - \\
Auxiliar de Primera & 8 & 8,7 & 7 & 1 & - \\
Total & 92 & 100 & & & \\
\hline
\end{tabular}

Fuente: Elaboración propia

La baja respuesta del profesorado coincide con estudios anteriores como el de Antón para el total del profesorado de la Universidad de Alicante, el realizado por Guil, Solano y Álvarez para el total de la Comunidad Andaluza; el de Matus y Gallego para la Universidad Pablo de Olavide o el de Tomás y Guillamón para cuatro universidades catalanas. Además, las conclusiones de éstos apuntan hacia la existencia de barreras y obstáculos, así como hacia la invisibilización y negación del profesorado, coincidiendo con los resultados de esta investigación. 


\subsection{Método}

El método utilizado fue la encuesta simple, diseñada y validada anteriormente a su envío por expertas en la materia durante el mes de mayo de $2016^{2}$. Ésta fue enviada en tres ocasiones a través de correo electrónico por parte del Consejo de Investigación y del Centro de Investigaciones Sociales y Educativas del Norte argentino (CISEN) de la Universidad Nacional de Salta (UNSa), donde se adjuntaba una carta solicitando la participación del cuerpo docente y desarrollando los objetivos del estudio.

En el cuestionario se combinaron varias técnicas: pregunta cerrada dicotómica y categorizada, la Escala de Likert y la pregunta abierta. De este modo podemos diferenciar cinco partes en el cuestionario:

1. Presentación y objetivos del estudio

2. Datos de control sobre las características sociodemográficas de la muestra (sexo, edad, dedicación, área académica, categoría laboral, antigüedad en el acceso y en la categoría actual)

3. Preguntas con respuestas cerradas dicotómicas y categorizadas (8 preguntas a través de las cuáles se pretende conocer directamente la percepción de las personas encuestadas acerca de la existencia o no de discriminación de género en la Universidad tanto en el acceso como en la promoción, así como en el mercado de trabajo en general, y otras cuestiones relacionadas con la participación de las mujeres en la Academia)

4. Un listado de 15 aseveraciones en base a una Escala Likert (se profundiza en la valoración que los/as encuestados/as realizan sobre los diversos tipos de barreras y obstáculos de género en la carrera académica)

5. Pregunta abierta donde se invita a expresar libremente cualquier tipo de comentario u opinión acerca del tema tratado.

Por último, los datos cuantitativos obtenidos de los cuestionarios fueron procesados y analizados a través del programa estadístico SPSS durante el último trimestre de 2016.

2. Intervinieron durante esta fase la Dra. Lina Gálvez, la Dra. M. ${ }^{a}$ del Carmen Monreal y el Dr. Mauricio Matus, pertenecientes a la Universidad Pablo de Olavide (Sevilla), junto con la profesora Dña. Gabriela Cecilia Barrios de la Universidad Nacional de Salta (Argentina). 


\subsection{Limitaciones}

Como principal limitación metodológica de la encuesta cabe señalar la tasa de no respuesta por parte del profesorado, lo que produce que la muestra no sea representativa, por lo que los resultados deben ser considerados con restricciones. A pesar de ésto, esta situación puede ser interpretada como un indicador de la baja o alta sensibilidad por parte de los diferentes grupos de los/as docentes que han respondido o no.

Por otro lado, al no poder implementar una muestra aleatoria, se puede suponer que las personas que han respondido a la encuesta son aquellas que sienten mayor motivación a expresar su opinión positiva o negativa al respecto o que poseen un mayor interés o motivación por los temas de género e igualdad. También es posible que una persona haya respondido dos veces a la encuesta, aunque no hay evidencia empírica para sospechar que esto ha ocurrido. En cualquier caso, la evidencia presentada es un aporte que se complementa al marco teórico y aplicado de anteriores investigaciones, además, la encuesta puede ser tenida en cuenta como un pretest para futuros estudios.

\section{DISCRIMINACIÓN DE GÉNERO EN EL SISTEMA UNIVERSITARIO DE ARGENTINA}

El sistema educativo universitario en Argentina se caracteriza por su feminización. Las cifras estadísticas son contundentes. Según la Secretaría de Políticas Universitarias de la Nación (SPU), para el 2014 -último año con datos disponibles-, en los estudios de pregrado y grado de las universidades argentinas las mujeres eran mayoría (57,2\% de matrículas y $61,5 \%$ de egresos). De igual forma, su participación era más elevada que la de los hombres en las maestrías ( $53,5 \%$ de las matrículas y $49,7 \%$ de egresos). Esta tendencia se continua manteniendo estable incluso entre los/as estudiantes de doctorado, donde el porcentaje de éstas constituían el 56,4\% del total.

Sin embargo, si atendemos a la distribución del alumnado según ramas de estudio, el panorama es distinto. Mientras que en campos como las Ciencias de la Salud o Ciencias Humanas en grado y pregrado las mujeres representaban el 73\% y el 69,4\% del total de matriculaciones, respectivamente, en Ciencias Aplicadas -donde se incluyen carreras como las ingenierías o informática- el porcentaje caía hasta el 36,3\% (SPU, 2014).

Si pasamos a analizar el cuerpo docente (preuniversitarios, universitarios y otros) del total de Universidades Nacionales, según los últimos datos disponibles del Sistema de Recursos Humanos de Universidades Nacionales (RHUN), para el año 2013 estaba formado por un total de 193.316 cargos, de 
los cuales 97.954 estaban ocupados por mujeres $(50,7 \%)$ y 95.632 por hombres (49,3\%). Entre los/as docentes del nivel preuniversitario se encontraba la mayor presencia de mujeres, el 63,3\% del total. Atendiendo al total de cargos docentes únicamente de nivel universitario, el número de éstos era 162.843, y el porcentaje de mujeres disminuía hasta el 48,9\% (Sistema RHUN).

La composición por sexo de las diferentes categorías académicas se reproduce en un gráfico «tijera» (Figura 1). Se puede apreciar una importante pérdida del potencial femenino tras la finalización de la tesis doctoral. Se pasa de una mayoría femenina entre los/as docentes de nivel preuniversitario y en la lectura de tesis doctoral, con un 57,6\% (SPU), a una representación de menos de la mitad del total de docentes universitarios/as, con una caída de más de diez puntos porcentuales. De este modo, éstas pasan de configurar el $48,9 \%$ del total de docentes universitarios/as al $45,4 \%$ del profesorado adjunto. Si avanzamos un escalafón más, el porcentaje cae hasta el 40,2\% del total del profesorado asociado/a. Finalmente, en la cúspide de la pirámide, la cifra de hombres titulares casi duplicaban a las mujeres: el 38,8\% del total de cargos titulares estaban ocupados por las mujeres y el $61,2 \%$ por hombres (Sistema RHUN). Se hace patente la existencia de un techo de cristal en la Universidad, que obstaculiza que las mujeres pueden alcanzar los estratos más altos de la jeraquía académica en igualdad de oportunidades que sus compañeros varones.

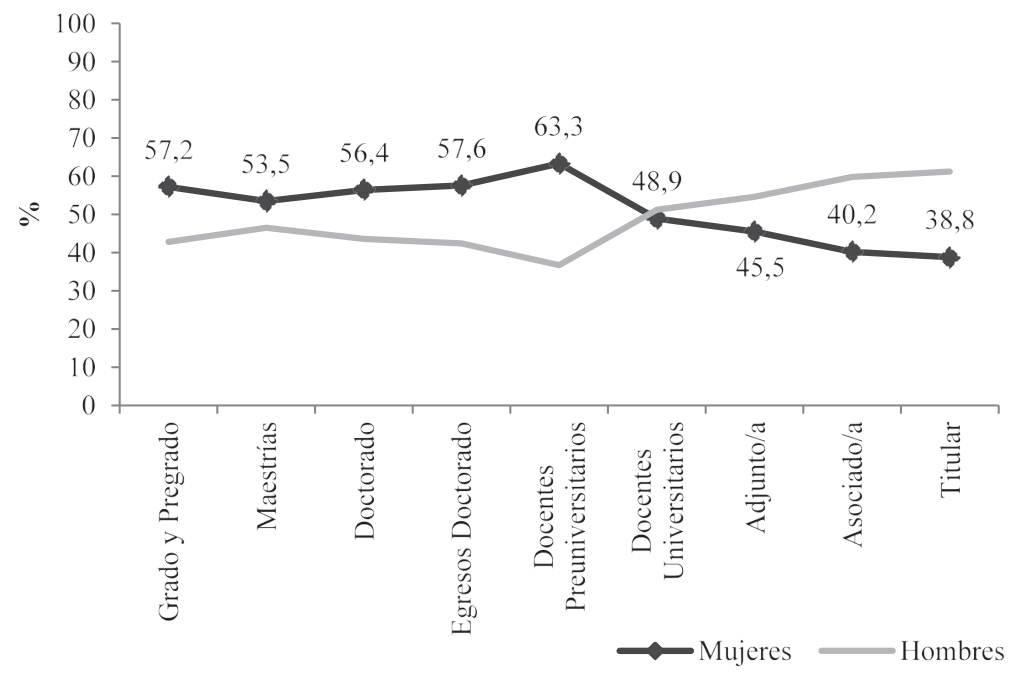

Figura 1. Distribución de hombres y mujeres en la carrera académica de las Universidades Nacionales. Años 2013-2014

Fuente: Elaboración propia sobre datos del SPU (2014) y Sistema RHUN (2013) 
Al analizar la participación del total de mujeres docentes universitarias argentinas entre los diferentes cargos y categorías académicas para el año 2013, encontramos que éstas se distribuían del siguiente modo: más de la mitad del total formaba parte de los/as auxiliares docentes (66,3\%), mientras que sólo 3,3 de cada 10 mujeres docentes eran profesoras, el 33,7\% (57,5\% y 42,5\% en el caso de los varones, respectivamente). Se produce una mayor presencia de las docentes entre los puestos bases (auxiliares docentes), al igual que entre los/as docentes preuniversitarios/as (Figura 2). De este modo, el porcentaje de mujeres profesoras sobre el total de mujeres docentes es superado en casi nueve puntos por los profesores varones. Se manifiesta la existencia de los «suelos pegajosos» (sticky floor) en el ámbito académico y científico -al igual que se produjera en el mercado laboral-, y que muestran, además, las dificultades de las mujeres incluso para acceder a los primeros niveles de la carrera académica (Torres y Pau).

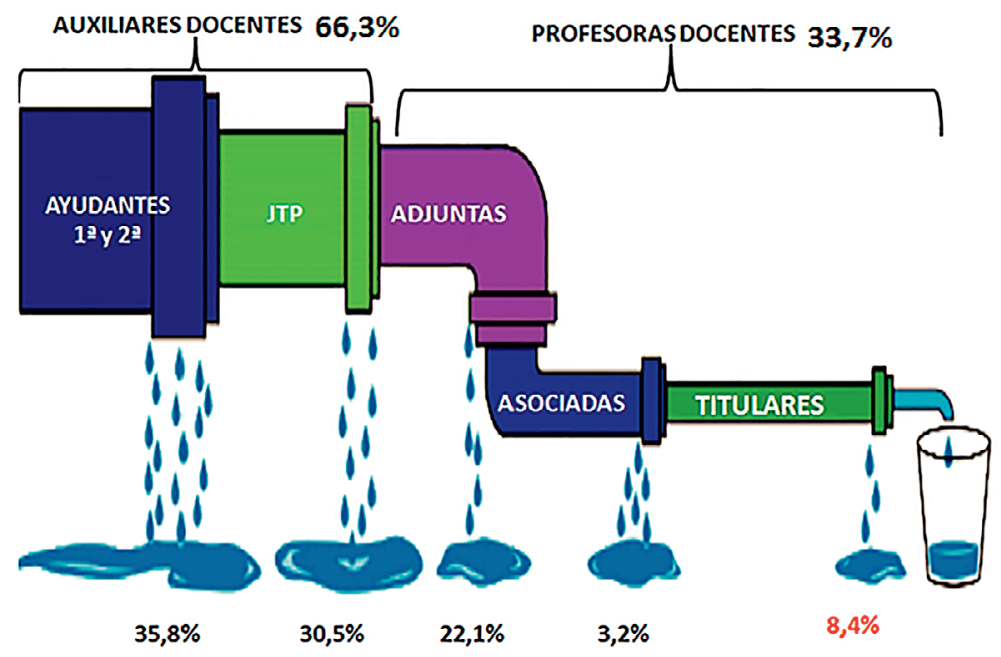

Figura 2. Distribución mujeres por cargos docentes en las Universidades Nacionales clasificados por categoría. Año 2013

Fuente: Elaboración propia a partir de los datos de Sistema RHUN (2013)

Entre las escalas de cada categoría, el 35,8\% de las mujeres docentes se agrupaban en los puestos bases de auxiliares (5,9\% auxiliares de segunda y 29,9\% auxiliares de primera), mientras que en las jefas de trabajos prácticos (JTP) éstas representaban el 30,5\%. Los hombres auxiliares, por su parte, constituían el 31\% del total de hombres docentes (7\% auxiliar de primera y 24\% de 
segunda) y el $26,5 \%$ de JTP. Las cifras van cayendo a medida que se va avanzando en la escala jerárquica, de modo que, entre los cargos del profesorado, el $22,1 \%$ del total de mujeres eran profesoras adjuntas y el 3,2\% profesoras asociadas ( $25,3 \%$ y $4,6 \%$ de hombres, respectivamente). Con respecto a la presencia de mujeres titulares, únicamente el $8,4 \%$ del total ocupaba este cargo, frente al 12,7\% de sus compañeros varones (Sistema RHUN).

Tal y como se desprende de los datos estadísticos, metafóricamente parece que existiera un «tubo que pierde» (leaky pipeline) o, también denominada tesis de la «pipeta», haciendo referencia a la pérdida del potencial femenino según se asciende de categoría o cargo, ya que «a medida que avanza el flujo a lo largo de la pipeta surgen barreras de diferente naturaleza que producen la exclusión de las mujeres» (Estébanez, De Filippo y Serial 9).

Considerando la presencia de la mujer en los puestos de responsabilidad entre las autoridades superiores de las Universidades Nacionales la situación no es mucho más esperanzadora. Al igual que ocurriera en la carrera académica, se produce una presencia mayoritaria de hombres en los principales órganos de gobierno y representación en la Universidad, responsables directos de la toma de decisiones en la misma (Figura 3).

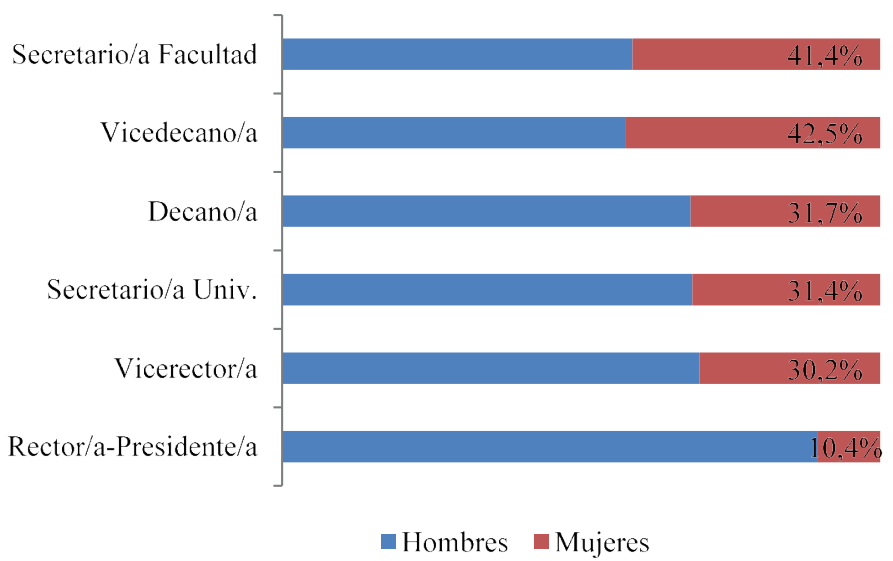

Figura 3. Cargos clasificados por categoría y sexo, total Universidades Nacionales.

Escalafón de Autoridades Superiores. Año 2013

Fuente: Elaboración propia sobre datos del Sistema RHUN (2013)

En el año 2013, del total de las autoridades superiores de las Universidades Nacionales las mujeres representaban solamente el 39,7\%, frente al 60,3\% de hombres. Éstas aparecen agrupadas en su mayoría en los vicedecanatos (42,5\%) 
y secretarías de facultad $(41,4 \%)$. No obstante, los porcentajes comienzan a descender hasta el 31,7\% de decanatos y el 31,4\% de secretarías de Universidad. El porcentaje de vicerrectoras era similar a los correspondientes a los otros cargos $(30,2 \%)$. Sin embargo, si atendemos al puesto de mayor poder en la Academia, el de rectora/presidenta, la representación de éstas disminuye hasta el 10,4\%. En términos absolutos, se traduce en la existencia únicamente de cinco mujeres rectoras en un total de 50 Universidades Nacionales.

\section{RESULTADOS}

En este apartado se presentan los principales resultados obtenidos, desagregados por sexo, y divididos en dos bloques principales. En primer lugar, se profundiza en la percepción que hombres y mujeres poseen de la discriminación de género en el mercado de trabajo, en general, y en la Universidad, en particular. En segundo lugar, se analiza la valoración que las personas encuestadas otorgan a las diferentes barreras y obstáculos existentes durante los procesos de promoción de la carrera académica e investigadora.

\subsection{Negación e invisibilización de la discriminación de género}

En primer lugar, se consultó acerca de la existencia o no de un mayor número de barreras para las mujeres que para los hombres en el mercado de trabajo argentino a la hora de alcanzar las categorías profesionales más altas. El 63\% de las personas consideran que las mujeres deben superar más obstáculos durante su trayectoria laboral. En el caso de las mujeres, el porcentaje de respuestas afirmativas aumenta hasta el 67,3\%. Sin embargo, más de la mitad de los hombres, el $52 \%$, niegan que las dificultades para las mujeres sean mayores que para ellos (Tabla 7 ).

Tabla 7. Opinión sobre la existencia o no de discriminación de género en el mercado de trabajo

\begin{tabular}{|l|c|c|c|c|}
\hline & HOMBRE & MUJER & N.d & TOTAL \\
\hline SI & $48 \%$ & $67,3 \%$ & $75 \%$ & $63 \%$ \\
\hline NO & $52 \%$ & $23,6 \%$ & $25 \%$ & $31,5 \%$ \\
\hline NS/NC & - & $9,1 \%$ & - & $5,4 \%$ \\
\hline
\end{tabular}

Fuente: Elaboración propia

Existe una tendencia por parte de las personas encuestadas a considerar la Universidad como un espacio más igualitario que el mercado de trabajo (63\%). 
Esta mayor sensación de igualdad por parte de los/as docentes puede deberse a la existencia de procesos de selección y promoción que se presuponen objetivos, por lo que hace pensar que la meritocracia opera en la Universidad con más intensidad que en otros sectores en general. Sin embargo, son los hombres aquellos que en mayor medida respondieron afirmativamente a esta pregunta en el $68 \%$ de los casos, frente al $56,4 \%$ de sus compañeras. Éstas, a su vez, en un $30,9 \%$ afirman que ambos espacios, tanto el mercado de trabajo como la Universidad son igual de meritocráticos ( $16 \%$ de hombres). Únicamente el $10,9 \%$ de mujeres y el $12 \%$ de hombres piensan que la igualdad es mayor en el mercado laboral (Tabla 8).

Tabla 8. Opinión sobre si la Universidad se constituye como un sistema más meritocrático que el mercado de trabajo

\begin{tabular}{|l|c|c|c|c|}
\hline & HOMBRE & MUJER & N.d & TOTAL \\
\hline Sí & $68 \%$ & $56,4 \%$ & $83,3 \%$ & $63 \%$ \\
\hline NO, más el trabajo & $12 \%$ & $10,9 \%$ & $8,3 \%$ & $10,9 \%$ \\
\hline NO, igual ambos & $16 \%$ & $30,9 \%$ & $8,3 \%$ & $23,9 \%$ \\
\hline NS/NC & $4 \%$ & $1,8 \%$ & - & $2,2 \%$ \\
\hline
\end{tabular}

Fuente: Elaboración propia

En línea con la creencia mayoritaria acerca de que la Universidad se constituye como un espacio más meritocrático que el mercado laboral, y tal y como la literatura revisada apunta, se produce una invisibilidad de la discriminación de género en la Universidad por parte del cuerpo docente. El 67,4\% del total de encuestados/as dicen no existir discriminación directa o indirecta hacia la mujer en el acceso a la carrera docente e investigadora en la Universidad Nacional de Salta (UNSa) (Tabla 9). El porcentaje cae en 7,4 puntos cuando se trata de negar la discriminación durante la promoción (62\%) (Tabla 10). De este modo, el porcentaje de personas que confirman que las mujeres sí se enfrentan a procesos discriminatorios cuando desean ascender a las categorías profesionales más altas es mayor que el obtenido con respecto a la discriminación en el acceso (32,6\% y 25\%, respectivamente).

$\mathrm{Si}$ atendemos a las diferencias por sexo, se observa que mientras que las mujeres son más conscientes de la existencia de la problemática durante la promoción $(34,5 \%)$ que durante el acceso $(29,1 \%)$, en el caso de los hombres la valoración se da a la inversa ( $16 \%$ de discriminación en el acceso y $12 \%$ en la promoción). Llama la atención que las propias perjudicadas, las mujeres, 
nieguen en un 60\% (acceso) y un 56,4\% (promoción) tal problemática. La negación por parte de los hombres docentes es aún más elevada en ambos casos, 8 de cada 10 hombres no creen que exista discriminación.

Tabla 9. Opinión sobre la existencia o no de discriminación de género en el acceso en la UNSa

\begin{tabular}{|l|c|c|c|c|}
\hline & HOMBRE & MUJER & N.d & TOTAL \\
\hline SI & $16 \%$ & $29,1 \%$ & $25 \%$ & $25 \%$ \\
\hline NO & $84 \%$ & $60 \%$ & $66,7 \%$ & $67,4 \%$ \\
\hline NS/NC & - & $10,9 \%$ & $8,3 \%$ & $7,6 \%$ \\
\hline
\end{tabular}

Fuente: Elaboración propia

Tabla 10. Opinión sobre la existencia o no de discriminación de género en la promoción en la UNSa

\begin{tabular}{|l|c|c|c|c|}
\hline & HOMBRE & MUJER & N.d & TOTAL \\
\hline SI & $12 \%$ & $34,5 \%$ & $66,7 \%$ & $32,6 \%$ \\
\hline NO & $88 \%$ & $56,4 \%$ & $33,3 \%$ & $62 \%$ \\
\hline NS/NC & - & $9,1 \%$ & - & $5,4 \%$ \\
\hline
\end{tabular}

Fuente: Elaboración propia

Como puede observarse en la tabla 11, no existe una opción preferente a la hora de valorar la presencia femenina en los puestos de responsabilidad de la UNSa. En mayor proporción los/as docentes consideran que ésta es acorde con el total del profesorado (35,9\%), seguidos/as por quienes afirman que son bastantes las mujeres que asumen cargos importantes, en un 32,6\%. Sin embargo, el 23,9\% creen que es muy poca. En las dos respuestas de los extremos, «mucha»y «muy poca», los porcentajes disminuyen hasta el $5,4 \%$ y el $1,1 \%$, respectivamente.

Hombres y mujeres muestran opiniones encontradas. Son los hombres aquellos que se encuentran más conformes con la participación actual de las mujeres en altos cargos, de modo que más de la mitad de éstos, el 52\%, afirman que su presencia es acorde con el total del profesorado $(29,1 \%$ en el caso de las mujeres). Entre aquellos/as que consideran que son bastantes las mujeres en estos puestos, se encuentra el 30,9\% de las mujeres y el 32\% de los hombres. Podemos afirmar que aunque son las docentes las más críticas y quienes muestran un mayor descontento ( $30,9 \%$ poca y $1,8 \%$ muy poca), son, a su vez, 
las que seleccionaron en mayor medida la respuesta «mucha», con 5,5\% (1,5 puntos porcentuales por encima de los hombres).

Tabla 11. Opinión sobre la presencia femenina en puestos de responsabilidad en la UNSa

\begin{tabular}{|l|c|c|c|c|}
\hline & HOMBRE & MUJER & N.d & TOTAL \\
\hline MUCHA & $4 \%$ & $5,5 \%$ & $8,3 \%$ & $5,4 \%$ \\
\hline BASTANTE & $32 \%$ & $30,9 \%$ & $41,7 \%$ & $32,6 \%$ \\
\hline ACORDE AL TOTAL & $52 \%$ & $29,1 \%$ & $33,3 \%$ & $35,9 \%$ \\
\hline POCA & $12 \%$ & $30,9 \%$ & $16,7 \%$ & $23,9 \%$ \\
\hline MUY POCA & - & $1,8 \%$ & - & $1,1 \%$ \\
\hline NS/NC & - & $1,8 \%$ & - & $1,1 \%$ \\
\hline
\end{tabular}

Fuente: Elaboración propia

\subsection{Barreras y obstáculos de la carrera académica}

Uno de los resultados más interesantes lo constituye la opinión de los/as docentes cuando se les pregunta acerca de si se han encontrado o no con barreras y dificultades para promocionar a lo largo de su carrera académica. Paradójicamente, a pesar de que la gran mayoría de éstos negaron la existencia de procesos de discriminación directos o indirectos durante la promoción, es decir, el techo de cristal, más de la mitad de las personas encuestadas confirman haber tenido que superar obstáculos para poder acceder a las categorías más altas, el 57,6\% (Tabla 12). No obstante, no existen diferencias notables por sexos, aunque el porcentaje de mujeres que respondieron afirmativamente $(54,5 \%)$ es escasamente superior al de hombres (52\%).

Tabla 12. Opinión sobre la existencia o no de barreras y dificultades para promocionar

\begin{tabular}{|l|c|c|c|c|}
\hline & HOMBRE & MUJER & N.d & TOTAL \\
\hline SI & $52 \%$ & $54,5 \%$ & $83,3 \%$ & $57,6 \%$ \\
\hline NO & $44 \%$ & $43,6 \%$ & $16,7 \%$ & $40,2 \%$ \\
\hline NS/NC & $4 \%$ & $1,8 \%$ & - & $2,2 \%$ \\
\hline
\end{tabular}

Fuente: Elaboración propia 
Por otro lado, cuando se les consulta si creen que las barreras y obstáculos que han tenido que ir superando a lo largo de su trayectoria profesional afectan de igual manera a sus compañeros/as del sexo puesto, el 54,3\% considera que no. Analizando la variable de género apenas se producen diferencias entre las respuestas de ambos sexos. Tanto mujeres como hombres, en mayor medida, niegan que las dificultades sean iguales para unos y otras ( $56 \%$ de hombres y $56,4 \%$ de mujeres). No obstante, los varones consideran en casi 6 puntos más que las mujeres $(30,9 \%)$ que la superación de obstáculos es igual para sus compañeras que para ellos. Especificar que en esta cuestión un elevado porcentaje de mujeres seleccionaron la respuesta no sé/no contesto, el 12,7\%, configurándose como la pregunta que más duda ha provocado entre las personas encuestadas, hombres y mujeres (Tabla 13).

Tabla 13. Consideración acerca de la influencia o no de las barreras en los compañeros/as del sexo opuesto

\begin{tabular}{|l|c|c|c|c|}
\hline & HOMBRE & MUJER & N.d & TOTAL \\
\hline SI & $36 \%$ & $30,9 \%$ & $58,3 \%$ & $35,9 \%$ \\
\hline NO & $56 \%$ & $56,4 \%$ & $41,7 \%$ & $54,3 \%$ \\
\hline NS/NC & $8 \%$ & $12,7 \%$ & - & $9,8 \%$ \\
\hline
\end{tabular}

Fuente: Elaboración propia

Finalmente, se les presentó un listado con 15 aseveraciones, basado en una escala Likert, en el que se les requería que valorasen de 1 (menos incidencia) a 4 (más incidencia) las barreras y obstáculos que aparecían recogidos según consideraban afectaban en mayor o menor medida a la hora de promocionar en la carrera académica. Como se puede observar a continuación, y para facilitar la comprensión y el análisis de los resultados, se diferencian tres tipos de barreras (personales, organizacionales y del contexto social), siguiendo la clasificación realizada por Guil (93):

\section{Barreras personales:}

1.1. Familiares:

1.1.1. Problemas de conciliación debido a la inexistente corresponsabilidad por parte de la pareja

1.1.2. Trabajo doméstico y de cuidados (hijos/as, personas dependientes)

1.2. Profesionales:

1.2.1. Doble jornada laboral 
1.3. Cognitivo-emocionales:

1.3.1. Baja autoestima y autoimagen

1.3.2. Falta de confianza y miedo

1.3.3. Auto-restricción (no promoción por voluntad propia)

1.3.4. Sensación de culpa

\section{Barreras organizacionales:}

2.1. Discriminación directa por parte del profesorado y/o universidad

2.2. Existencia de redes informales de poder

2.3. Existencia de sistemas de cooptación

2.4. Diferenciación en la valoración de méritos según el sexo

2.5. Legislación vigente

2.6. Falta de apoyo y financiación

3. Barreras del contexto social:

3.1. Sociedad patriarcal

3.2. Estereotipos y roles de género

Como se recoge en la Figura 4, en primer lugar, el resultado más significativo consiste en la mayor valoración por parte de las mujeres en comparación con los hombres de todas las barreras, a excepción de aquellas que hacen referencia a los obstáculos personales cognitivo-emocionales, como la baja autoestima y autoimagen y la falta de confianza y miedo. Ambas fueron levemente más valoradas por los hombres ( 2 y 1,9 , respectivamente). Por otro lado, el obstáculo que mayor puntuación alcanzó fue aquel que aludía a la existencia de redes informales de poder, tanto por parte de los hombres como de las mujeres $(2,8)$.

Las barreras que obtuvieron una mayor puntuación por parte de las mujeres, son aquellas que se producen en el contexto social, como la existencia de una sociedad patriarcal $(2,6)$ con unos arraigados estereotipos y roles de género $(2,5)$, junto con barreras organizacionales como la falta de apoyo y financiación $(2,6)$ y la existencia de sistemas de cooptación $(2,5)$. De igual modo, las barreras personales familiares como el trabajo doméstico y de cuidados y la doble jornada laboral fueron puntuados con 2,5.

En el caso de los hombres, no se obtienen resultados que difieran considerablemente de los de las mujeres. Así, los obstáculos que según éstos tienen una mayor incidencia a la hora de promocionar son coincidentes con los de sus compañeras: las barreras organizaciones como son los sistemas de cooptación $(2,5)$ y la falta de apoyo y financiación $(2,3)$. Resulta paradójico que a pesar de lo que muestran las encuestas de usos del tiempo, es decir, que el trabajo no remunerado de cuidados y domésticos son asumidos casi en exclusividad por las mujeres, esta situación y las consecuencias que de ésta se desprenden, 
la doble jornada laboral, sea considerada por los hombres como una dificultad en su camino hacia la cúspide de la pirámide jerárquica, con 2,4 puntos.

La principal diferencia entre sexos se encuentra en la valoración de las causas que emergen del contexto social, fuertemente valoradas por las mujeres, mientras que los hombres le asignan 2 puntos de media. De igual modo, con medio punto de diferencia, se encuentra el sesgo de género en la valoración del mérito (1,6 los hombres y 2,1 las mujeres) y, en la línea de las respuestas anteriores, la discriminación directa por parte del profesorado y/o la universidad. Aunque se continúa produciendo una negación e invisibilización por parte de los hombres docentes (1,6 puntos), las mujeres le otorgan una puntuación de 2,1.

Finalmente, aquellas barreras menos valoradas por ambos sexos fueron la sensación de culpa, la auto-restricción, y la normativa vigente. En el caso de la auto-restricción nos resulta interesante detenernos a comentar la baja puntuación que las mujeres han otorgado, sólo 1,6 puntos. Esto desmonta, así, la falacia de la libre elección, a través de la cual se argumenta la auto-exclusión voluntaria de las mujeres de los procesos de promoción, al priorizar su vida familiar por encima del desarrollo profesional. Este argumento es utilizado para explicar las bajas cifras de participación de las mujeres en los círculos de poder universitarios.

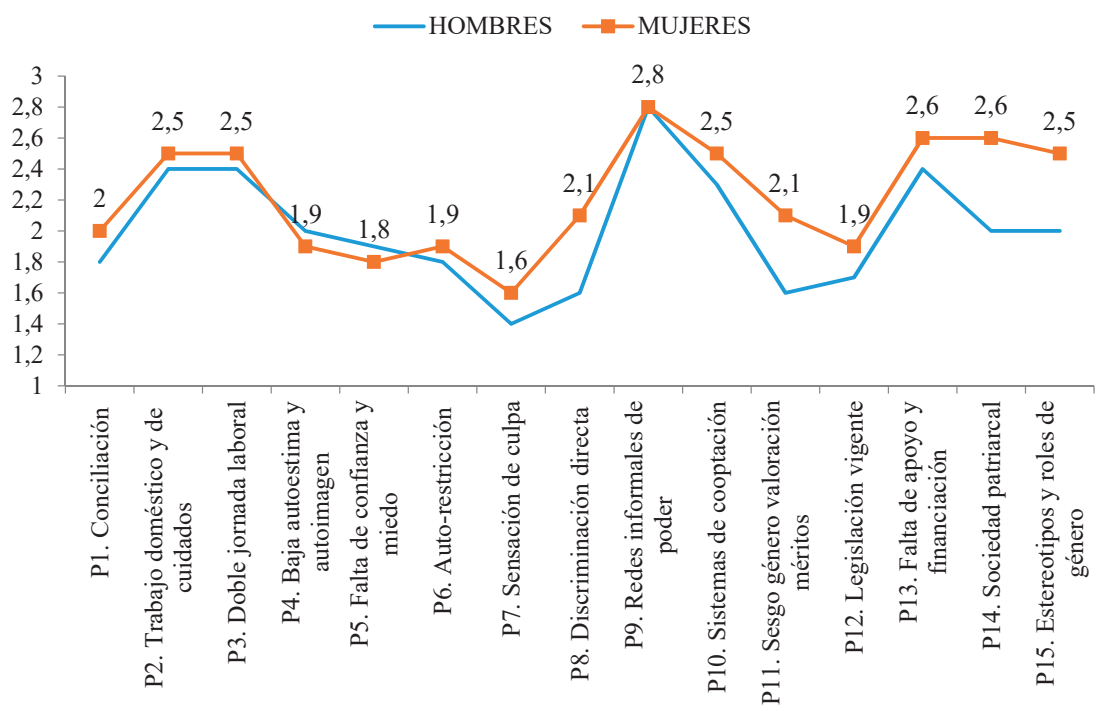

Figura 4. Valoración de las barreras y obstáculos de promoción

Fuente: Elaboración propia 


\section{DISCUSIÓN Y CONCLUSIONES}

Este trabajo ha permitido realizar una primera aproximación a las percepciones y opiniones que el cuerpo docente de la Universidad Nacional de Salta posee acerca de la discriminación de género en el contexto académico. Hemos podido comprobar cómo las cifras estadísticas son claras, mostrando un panorama de desigualdad. Por un lado, se produce una acumulación de mujeres en aquellas áreas para las que son consideradas «aptas», debido a que se constituyen como una extensión de los roles de cuidados y familiares que han asumido tradicionalmente (segregación horizontal).

Por otro lado, y a pesar de que representan una mayoría entre el total del cuerpo docente, éstas son retenidas por los suelos pegajosos, encontrándose sobrerrepresentadas en aquellos puestos bases, como es la categoría de docentes auxiliares. A todo ello se le suma el leaky pipeline, es decir, el descenso en la participación a medida que se avanza hasta los cargos jerárquicos más altos, produciéndose una fuga del potencial femenino y constatándose la existencia del techo de cristal.

En primer lugar, tal y como la literatura ha mostrado (Guil, Solano y Álvarez; Matus y Gallego; Pastor, Belzunegui, Moreno y Mañas; Pérez, Santesmases y Alcalá), podemos confirmar que se produce una invisibilización del problema. Se trata de una negación, además, que es más fuerte por parte de los hombres. Éstos niegan la existencia de cualquier tipo de discriminación de género tanto en el mercado laboral como en la Universidad. En todas las ocasiones en las que son consultados a lo largo del cuestionario, invisibilizan en mayor medida que sus compañeras la situación de desigualdad en la que se encuentran las mujeres, la escasa representación de éstas en los altos cargos y puestos de responsabilidad y poder, y otorgan valores más bajos a todas las barreras y obstáculos del techo de cristal.

Esta situación puede ser explicada debido a que los hombres poseen una conciencia del estigma del género más baja que las mujeres. En una investigación realizada por Pinel, ésta demostró que ciertos grupos, como en este caso las mujeres, poseen una mayor conciencia del estigma, por lo que esperan ser estereotipadas por el hecho de ser mujeres. Además, mientras más alto es el grado de conciencia, mayor es la probabilidad de que los estereotipos y prejuicios de género sean identificados y rechazados.

Los resultados de las encuestas muestran que al contextualizar la discriminación de género en el espacio académico, más de la mitad de los/as consultados/as responden negando la evidencia. Sin embargo, el porcentaje de mujeres que confirma su existencia es mayor durante el proceso de promoción que en el momento del acceso (en los hombres se produce a la inversa). Este 
resultado es coherente si tenemos en cuenta que el estudio ha sido realizado en una universidad con una alta tasa de feminización. De igual modo, la mayor parte cree que la presencia de las mujeres en los puestos de responsabilidad es adecuada al total del profesorado, siendo los hombres los que muestran una mayor satisfacción al valorar dicha presencia.

Por otro lado, la tendencia general consiste en considerar la Universidad como un espacio más meritocrático que el mercado laboral. De este modo, la gran mayoría de mujeres afirma que los obstáculos que deben superar para promocionar en el mercado de trabajo son mayores, sin embargo, más de la mitad de éstas continúan sin aceptar que se produzca ningún tipo de desigualdad en la Universidad.

En segundo lugar, paradójicamente, aunque la discriminación directa o indirecta es negada, al formular la pregunta de un modo más personal y directo, solicitándoles que consideren si se han tenido que enfrentar a obstáculos y barreras para promocionar a lo largo de su carrera, el número de personas que responden afirmativamente se convierte en mayoría. Así, la existencia de redes informales de poder es valorado como el principal obstáculo en las carreras de ambos sexos.

Se trata de redes implícitas de poder contra las que difícilmente se puede luchar (Guil 24), precisamente por la sutileza con la que actúan. En palabras de Ballarín, se configuran como un «entramado de relaciones masculinas donde se sostiene el poder que legitima la teoría» (184). Es por ello que no es casual que sea una de las causas más valorada por las docentes, sin embargo, llama la atención la alta puntuación proporcionada, además, por los hombres.

Finalmente, señalar que las mujeres valoran estas dificultades con mayores puntuaciones que sus compañeros varones, dándole especial importancia a las barreras que emergen del contexto social, como los estereotipos y roles de género en una sociedad patriarcal, junto a la doble jornada laboral (consecuencia de los trabajos domésticos y de cuidados) y, la falta de apoyo y financiación y los sistemas de cooptación, como barreras organizacionales.

En resumen, en este trabajo se ha constatado diferencias en las opiniones y percepciones de los hombres y mujeres docentes. La concienciación y visibilización de los procesos de discriminación de género, junto con el análisis y la profundización en las barreras y obstáculos se constituyen como una tarea fundamental. El diseño e implementación de medidas y estrategias de intervención difícilmente podrán obtener resultados positivos si las personas implicadas no consideran el problema como tal. 


\section{REFERENCIAS BIBLIOGRÁFICAS}

Antón, Susana. Informe Académicas en la Universidad de Alicante. Cuadernos de Trabajo de Investigación 12. Alicante: Universidad de Alicante, 2005.

Bailyn, Lotte «Academic careers and gender equity: Lessons learned from MIT». Gender, Work and Organization 10.2 (2003): 137-153.

Ballarín, Pilar. «Mujeres en el "laberinto de cristal" universitario». Miradas desde la perspectiva de género: estudios de las mujeres. Ed. Isabel de Torres. Madrid: Narcea Ediciones, 2005, 183-194.

Baxter, Janeen y Erik Olin Wright. «The glass ceiling hypothesis: A comparative study of the United States, Sweden, and Australia». Gender and Society 14.2 (2000): 275-294.

Busso, Matias y Dario Romero Fonseca. Female labor force participation in Latin America: Patterns and explanations. Ciudad de la Plata: Universidad Nacional de la Plata, Documentos de Trabajo del CEDLAS, 2015.

Cohen, Philip y Matt L. Huffman. «Individuals, Jobs and Labor Markets: The Devaluation of Women's Work», American Sociological Review 68.3 (2003): 443-463.

Comisión Europea. She Figures 2015. Gender in research and innovation. Bruselas:Dirección General de Investigación e Innovación, 2016. <https:// ec.europa.eu/research/swafs/pdf/pub_gender_equality/she_figures_2015-final. pdf>, consultado el 16-12-2016.

CTIO. Documento de trabajo para el debate en el marco de la CTIO - Género. Argentina: Ministerio de Trabajo, Empleo y Seguridad Social. Presidencia de la Nación, 2016. <http://www.trabajo.gov.ar/downloads/cegiot/Informe_CTIO_ DocumentoDeTrabajo.pdf>, consultado el 03-01-2017.

Estébanez, María Elina, Daniela De Filippo y Alejandra Serial. La participación de la mujer en el sistema de Ciencia y Tecnología en Argentina. Buenos Aires: Documento de Trabajo nro. 8. Proyecto GENTEC. Informe final Grupo REDES. UNESCO-OEI, 2003.

Fernández, Concepción. «La mujer en la Universidad española. Docencia, investigación y poder. Datos y aspectos cualitativos». Revista de Educación 290 (1989): 61-171.

Gallego, Nazareth. «Rompiendo el techo de cristal: la lectura de la tesis doctoral como momento clave.» Mètode: Revista de difusión de la Investigación 91 (2016): 64-71.

Gálvez, Lina y Paula Rodríguez. «El empleo de las mujeres en la España democrática y el impacto de la Gran Recesión», Áreas Revista Internacional de Ciencias Sociales 32 (2013): 105-123. 
Gálvez, Lina, Paula Rodríguez, Astrid Agenjo y Mónica Domínguez. El Trabajo de Cuidados de Mujeres y Hombres en Andalucía. Medición y valoración. Sevilla: Instituto Andaluz de la Mujer, 2013.

García de León, María Antonia. «Las profesoras universitarias: el caso de una élite discriminada». Revista Complutense de Educación 1.3 (1990): 355-372.

Gómez, Carmuca. «Mujeres y trabajo: principales ejes de análisis». Papers: revista de sociologia 63 (2001): 123-140.

Guil, Ana. Techos de Cristal en la Universidad Hispalense. Informe final del proyecto del Plan Nacional I+D+i 2001-2004. Sevilla: Universidad de Sevilla, 2005.

Guil, Ana, Ana Solano y Manuela Álvarez. La situación de las mujeres en las universidades públicas andaluzas. Sevilla: Consejo Económico y Social de la Junta de Andalucía, 2005.

Hartmann, Heidi. «Capitalismo, patriarcado y segregación de los empleos por sexos». Las mujeres y el trabajo. Rupturas conceptuales. Eds. Cristina Borderías, Cristina Carrasco y Carme Alemany. Madrid: Icaria, 1994, 253-294.

INDEC. Encuesta sobre trabajo no remuerado y uso del tiempo. Argentina: Ministerio de Trabajo, Empleo y Seguridad Social. Presidencia de la Nación, 2014. <http:// www.indec.gov.ar/uploads/informesdeprensa/tnr_07_14.pdf>, consultado el 04-01-2017.

INDEC. Encuesta Permanente de Hogares (EPH). Argentina: Ministerio de Trabajo, Empleo y Seguridad Social. Presidencia de la Nación, 2016. <http://www.indec. gob.ar/uploads/informesdeprensa/indicadores_eph_3trim16.pdf>, consultado el 05-01-2017.

Jackson, Jerlando F.L y Elizabeth M. O'Callaghan. «What do we know about glass ceiling effects? A taxonomy and critical review to inform higher education research». Research In Higher Education 50.5 (2009):460-482.

Matus, Mauricio y Nazareth Gallego. «Techo de cristal en la Universidad. Si no lo veo no lo creo». Revista Complutense de Educación 26.3 (2015): 611-626.

Monreal, María del Carmen y Belén Martínez. Esquemas de género y desigualdades sociales, en Intervención Social y Género. Madrid: Narcea Ediciones, 2010.

Morrison, Ann M., Randall White y Ellen Van Velsor. Breaking the Glass Ceiling: Can Women Reach the Top of America's Largest Corporations? New York: AddisonWesley, 1987.

Moser, Caroline y Kate Young. «Women of the working poor.» The IDS Bulletin 12.3 (1981): 1-9.

OEDE. Boletín de estadísticas de género y mercado de trabajo. Argentina: Ministerio de Trabajo, Empleo y Seguridad Social, 2014. <http://www.trabajo.gov.ar/left/ estadisticas/genero/estadisticas.asp>, consultado el 20-11-2016.

Ogbogu, Christina O. «Gender factors affecting female labour input in the Nigerian University System». Gender and Behaviour 8.1 (2010): 2666-2677. 
OIT. Las mujeres en el trabajo: Tendencias de 2016. Ginebra: Oficina Internacional del Trabajo, 2016. <http://www.ilo.org/wcmsp5/groups/public/---dgreports/--dcomm/---publ/documents/publication/wcms_483214.pdf>, consultado el 04-01-2017.

Pastor, Inma, Ángel Belzunegui, Blanca Moreno y Carmen Mañas. «La igualtat d'oportunitats a la universitat: les percepcions del PDI». Papers: revista de sociología 95.2 (2010): 457-481.

Pateman, Carol. El contrato sexual. Barcelona: Anthropos Editorial, 1995.

Pérez, Eulalia, Maria Jesús Santesmases y Paloma Alcalá (Coords.). Mujer y ciencia. La situación de las mujeres investigadoras en el sistema español de ciencia y tecnología. Madrid: Ministerio de Educación y Ciencia. Fundación Española para la Ciencia y Tecnología, 2005.

Pinel, Elizabeth C. «Stigma Consciousness: The Psychological Legacy of Social Stereotypes». Journal of personality and social psychology 76.1 (1999): 114-128.

PNUD. Aportes para el desarrollo humano en Argentina 2014. Género en el trabajo: brechas en el acceso a puestos de decisión. Buenos Aires: Programa de las Naciones Unidas para el Desarrollo, 2014. <http://www.ar.undp.org/content/dam/ argentina/Publications/Desarrollo\%20Humano/PNUD\%20ARGENTINA\%20_ Aportes_8.pdf>, consultado el 10-01-2017.

Probert, Belinda. «I just couldn't fit in: Gender and Unequal Outcomes in Academic Careers». Gender Work and Organization 12.1 (2005): 50-72.

Sarasúa, Carmen y Lina Gálvez. ¿Privilegios o eficiencia?: mujeres y hombres en los mercados de trabajo. Alicante: Universidad de Alicante, 2003.

Sistema RHUN. Área de Recursos Humanos de las Universidades Nacionales. Argentina: Dirección Nacional de Presupuesto e Información Universitaria, 2013.

SPU. Anuario de estadísticas universitarias de Argentina. Ciudad Autónoma de Buenos Aires: Ministerio de Educación de la Nación, 2013. <http://informacionpresupuestaria.siu.edu.ar/DocumentosSPU/Anuario_2013.pdf>, consultado el 10-07-2016.

SPU. Sistema de consultas de estadísticas universitarias. Argentina: Ministerio de Educación de la Nación, 2014. <http://estadisticasuniversitarias.me.gov.ar/\#/ homes, consultado el 12-07-2016.

Tomás, Marina y Cristina Guillamón. «Las barreras y los obstáculos en el acceso de las profesoras universitarias a los cargos de gestión académica». Revista de Educación 350 (2009): 253-275.

Torns, Teresa y Carolina Recio. «Desigualdades de género en el mercado de trabajo entre la continuidad y la transformación». Revista de Economía Crítica 14 (2012): 178-202. 
Torres, Obdulia y Bernadette Pau. «Techo de cristal y suelo pegajoso: La situación de la mujer en los sistemas alemán y español de ciencia y tecnología». CTS: Revista Iberoamericana de Ciencia, Tecnología y Sociedad 6.18 (2012): 35-59.

UNSa. Estadísticas de la planta docente de la Universidad Nacional de Salta. Ciudad de Salta: Departamento de Estadísticas Universitarias, 2015. 